\title{
2D- und 3D-Röntgen bei einfacher Bedienung
}

Auf der Plattform des bewährten ORTHOPHOS XG hat Sirona ein Röntgensystem mit innovativer Aufnahmetechnologie für höchste Zeichenschärfe bei geringstmöglicher Dosis entwickelt. Sharp Layer, eine Technologie, die die Panoramakurve des Sensors automatisch den individuellen anatomischen Eigenschaften des Patienten anpasst und scharf abbildet, ist das namensgebende Merkmal des neuen ORTHOPHOS SL. Die 2. Neuerung für eine Bildqualität auf komplett neuem Niveau ist der Direct-Conversion-Sensor(DCS). Er erzeugt aus den Röntgenstrahlen unmittelbar elektrische Signale und geht dabei nicht, wie bisher üblich, den Zwischenschritt über eine Umwandlung in Licht. Dadurch gehen weniger Informationen verloren und die Ausbeute an Bildinformationen ist bezogen auf die applizierte Dosis signifikant besser. Der Direct-Conversion-Sensor erzeugt so bei reduzierter Strahlendosis besonders effizient Röntgenbilder mit einer hohen Zeichenschärfe. Auch im 3D-Modus ermöglichen zahlreiche Verbesserungen wie zum Beispiel die Metallartefaktreduzierung, die Bildprozessierung und die Möglichkeit, den HD-Modus zuzuschalten, eine hervorragende Bildqualität. Dabei kann die Strah- lendosis durch die Auswahl von 3 verschiedenen Volumengrößen $(5 \times 5,5 \mathrm{~cm}$, $8 \times 8 \mathrm{~cm}, 11 \times 10 \mathrm{~cm}$ ) und Kollimierungen in Abhängigkeit der diagnostischen Fragestellung angepasst und minimiert werden. Das Gerät ist in einer aufrüstbaren 2D-Variante oder als 3D-Hybridgerät mit einem großen Volumen $(11 \times 10 \mathrm{~cm})$ oder einem auf die allgemeine Zahnarztpraxis optimierten Volumen von $8 \times 8 \mathrm{~cm}$ erhältlich.

\footnotetext{
Nach einer Pressemitteilung der Sirona Dental GmbH, A - Wals bei Salzburg Internet: www.sirona.com
} 